\title{
Review-Influence of Nanomaterials on Cellular Stress, and Cellular Behavior
}

\author{
Samant MP1, Lahir $\mathrm{YK}^{2 *}$, Chitre $\mathrm{AV}^{3}$, and Kale $\mathrm{A}^{4}$ \\ 2,3Department of Biophysics, University of Mumbai, India \\ ${ }^{1,4}$ School of Chemistry, University of Mumbai, India
}

Review Article

Volume 3 Issue 3

Received Date: September 25, 2018

*Corresponding author: YK Lahir, Department of Biophysics, University of Mumbai,

Published Date: October 18, 2018

Vidyanagari, Santa Cruz, (E), Mumbai-400098, India, Email: lahiryk@gmail.com

\section{Abstract}

The cellular responses to stress are a complex phenomenon. These have faced varied impacts of the advancements in biomolecular, cellular and biomedical fields that have taken place from time to time. Nanotechnology and nanomaterials have played their major roles in these advanced achievements. The interactions between cells and the various nanomaterials are obvious due to fabricated and/or engineered nanomaterials that are designed with special features to attain specific set targets. These nanomaterials are utilized in varied modes such as drug carriers, remedial agents of environmental aspects, biotechnological and biomedical processes, implants, biosensors etc. The implications of these wonder materials in any biosystems, industrial processes and products etc ensure their interactions with cells. Nanomaterials are also capable to induce cellular stress in biosystem as they are the components of the products used in daily life. There are numerous biomolecules and the cellular processes that are involved in intra and intercellular communications. The cellular communication is the prime functional aspect for cell survival. The interaction between the cells and the nanomaterials are likely to influence this cellular communications and other cellular processes. These features in all probabilities affect cellular responses. Cellular responses are protective and/ or rendering the affected cells prone to necrosis or cellular death. Various nanomaterials with different reactive affinities are bound to influence the cellular responses with respect to the stress. This reflects on the needs to evaluate and understand the mechanism involved during the process of cellular stress and the related behavior.

Keywords: Cellular Stress; Cellular Responses; Cellular Communication; Nanomaterials; Biophysical Factors; Nanobiointerface

\section{Introduction}

\section{Overview of Cellular Stress and Cellular Response}

Stress is the resultant impact of specific factors like molecular flux, induced mechanical forces due to the flow of body fluids, intrinsic and extrinsic biomolecular and cellular movements, change in the density of body fluids, flux of toxins or any xenobiotics which affect minor to major changes in the ambient cellular environment. The state of stress may be expressed as a change in size and shape of cell, molecular fluctuations, intra and extra 
cellular hydrostatic pressure, change in the cell organelles, deficient recourses, infection, etc. Under normal range of varied stress caused by sub acute exposure to stress (physical, biochemical etc, there are no drastic changes in the structural, functional cellular integrity but extreme conditions of stress resulted by sub chronic or chronic exposure lead to cellular disorganization in their structural and functional integrities. The degree of disorganization is proportional to the degree of stress. The stress can be either acute or chronic in nature. Cell under stress exhibits changes in molecular, biochemical, conformational and functional adaptations or adjustments to retain the specific cellular homeostatic state. The degree and the duration of stress are the deciding parameters of the varied cellular fluctuations and/or adaptabilities.

A basic functional principle is that when ever work is done energy is used resulting in some change/s or fluctuations and these are exhibited in the body. Sometimes these changes are very finite and can be recorded. These changes are in accordance with rate, intensity and efficacy of the stimuli. It may be envisaged that all responses are dependent on the time and length scale concept [1]. Atoms combine and form complex molecules that in turn form structural and functional domain of megamolecules or basic structural aspects of cell organelle and so on. When micro, macro and mega molecules of typical size and form move across the biological barrier i.e. cell membrane and associated structures, during translocation, transportation there is a physical and chemical impact on the barrier because these molecules exert physical stress on its finite constitutional component [2]. Thus a biological entity experiences osmotic pressure, turgor pressure, tonicity (osmotic pressure gradient), forces due to osmosis, viscosity of body fluids, and concentration gradient across the membrane under consideration and so on. The physical forces caused due to these conditions impact the biological barrier. Further, such zones of the cell membranes undergo physical stress along with their constitutional components [3]. Cell membrane whether prokaryotic or eukaryotic, either solitary or in groups, possess some of the physical properties such as elasticity, permeability, electrical potential with reference to charged molecules of the cell membrane or those moving across it.

Although cell is a structural and functional unit of a biosystem, it is influenced by the biophysical aspects. In biophysical terms it does work at the cost of some energy. Thus in addition to biochemical influence there are biophysical influences affecting the functional and regulatory aspects of cell depending on the degree of impact and type of the cell.

The regulatory role of cell includes (i) variety, nature and number of membranes, (ii) nature of lipid components associated with membrane, (iii) causing appropriate fluctuations in the components of glycocalyx such as glycocalyx protein and lipid associated carbohydrate molecules, (iv) regulatory aspects related to the selectivity of the integrated membrane proteins in order to permit these proteins to get transferred to the respective molecules bound along with them, (v) monitoring the single integral membrane protein and/or closely related or associated components to facilitate the movements to and fro across the membrane depending on the nature of the ions and other molecules, (vi) providing appropriate functional area for inter actions that involve folding, indenting, averting, getting pinched off etc, (vii) permitting the movements of molecules either embedded in membrane or present either on the inner or outer surfaces of the membrane [4]. A cell under stress responds to any reaction that causes distortion or damage to macromolecules beyond its threshold limits. The cellular response is focused temporarily to enhance the limits of tolerance towards the distortion of macromolecules. This is accomplished by utilizing the 'preserved phylogenetic set of gene' and the pathways involving stabilization of the macromolecule. The repair of damaged macromolecule is directed to the promotion of cellular and organismal integrity under non-optimal cellular state. The overall related mechanism needs specific time limit to sort out special stressor-specific adaptation that is designed to reestablish homeostatic conditions of the cell under stress [5]. Kultz tried to summarize cellular response to stress as (i) cellular response is related to changes caused in extra cellular factors that have damaged the structural and functional integrity of macromolecule/s, (ii) there is a specific set of homologous proteins that participate during cellular response in most of the biosystem, (iii) when cell is subjected to multiple stresses its response becomes synergistic in nature. When a cell is pre-exposed to one type of stress this stress in turn induces 'transient stresshardening'. This cell may be induced to develop 'cross tolerance' to other forms of stresses [5].

In general there are four major modes of cellular responses during adverse, abnormal and clinical or pathophysiological conditions. These modes functionally converge towards the stabilizing structural and functional integrity of macromolecules. These four modes also try to conserve metabolic energy to reestablish cellular homeostasis [5]. 
1. The check points of cell cycle regulate the growth. This cell cycle check point involves G1/S check point (Bartek and Inkas, 2001), G2/M check point and translational control mechanism [6].

2. Molecular chaperons (HSPs) and protein stabilizers get activated involving phosphorylation of chaperon (HSP28) through p38 MAP kinase signaling path ways [7].

3. Mechansim of stabilization, repair of nucleic acids and chromatin gets activated involving p53 pathway, and NFk $\beta$ path way [8].

4. The molecular debris formed during the stress period is cleared involving 'Ubiquitin-proteasome pathway'.

The processes like 'quality control of newly synthesized proteins', regulation of transcriptional factors, expression of genes, differentiation of cells, responses related to immune stimuli, pathogenic conditions like cancer, neurodegenerative defect and diseases, atherosclerosis, inflammatory process, formation of cataract, senescence, cell cycle, etc come under 'Cellular degradation system [9]. The efficacy of the biosystem is broadly related to its capability to cope with intrinsic and extrinsic stress. Genetic and extrinsic factors do have their roles in stress response mechanism. 'Ubiquitin-proteasome system' is the primary proteolytic mechanism that regulates the degradation of damaged proteins. It also controls the turn-over of the proteins of cytosol and nucleus. Basically two steps are involved during the degradation of proteins under ubiquitinproteasome system: in first step the protein to be degraded is tagged with polyubiquitin chain and during second step the tagged protein is degraded using proteasome. Ubiquitin is a conserved protein and has 76 amino acids. The tagging involves polyubiquitination; this process is complex and involves ubiquitin protein and three different enzymes [10]. The proteins to be degraded undergo a complex process called ubiquitination. Leestemaker and Ovaa mentioned that ubiquitination is accomplished in three steps. In first step ubiquitin gets activated involving enzyme E1 and ATP, in second step the activated ubiquitin is transferred to enzyme E2 and during third step the complex of ubiquitin and E2 is shifted to the lysine residue of the target protein. This shifting is dependent on the enzyme E3. The ubiquitinproteasome system plays a central role during establishing cellular homeostasis. The process of ubiquitination can be reversed when 'deubiquitination enzymes' are employed. During this process ubiquitin moiety is separated from the target protein. These deubiquitination enzymes are also able to trim the ubiquitin chain [11].
Two major parameters like degree of severity and the duration of the stress play deciding role during the stressed cellular behavior. During such conditions the cell either tries to retain its normal cellular homeostasis or tries to adapt to the conditions set by the stress on the cell. The cellular response may include (i) initiation of the repair mechanism using available resources, (ii) induction of temporary adaptive efforts with respect to the stressful conditions, (iii) may induce autophagy or (iv) initiation of process of cellular death [12]. Cellular repair mechanism involves change in gene expression, modulate the transcription under stressed conditions, controlled repair of quality protein, repair the damage caused by the stress or modify the response accordingly [12].

Intercellular communication is related to the nature of the respective signal i.e. contact mediated diffusible ligand; spatial distribution of signal and cell/cells; mode of reception of the signal. There is every chance that a signal may change with the change in the micro and/or ambient environment [13]. During the developmental phase the concerned mother cells show external ligand and the behavior is gradient based. This aspect establishes spatial pattern in the tissue and is accomplished either differentiating phenotype cells in graded manner or inducing the cellular movements towards the specific location. Such cellular behavioral movements are often observed among metastatic cells $[14,15]$. Cells exhibit response to chemicals that induce or act as stimuli or cues given by the extracellular matrix. These are responsible for chemo-toxicity. It is also dependent on the gradient cues or chemical gradient indication based on the specific substrata [16]. Individual cells give response with respect to external directed cues. These cues are spread spatially in the heterogeneously pattern. It may respond to the location of the cell. In case of immune response of cell i.e. leukocyte and macrophages, is also related to the graded cytokines released by the cell like endothelial cell. This enables the lymphocytes to reach the injured cell [17].

Cell under stress may modulate the signaling pathways like mammalian target of rapamycin, transformation of related protein-53, Necrotic factor kappa $\beta$, or may exhibit adaptive behavior with respect to the stress [18-22]. The stress can induce genomic instability or production of those proteins that are responsible for the protection of the cell or the disintegration of existing proteins and/or hinder their functions. The process of production of protective proteins is very complex in nature. The protective proteins (also called chaperons) have multiple functionalities. Their mode of function involves formation 
of sticky, carbon rich region of unfolded proteins referred as 'small pockets'. These also form a 'protective-arm' around the neighboring unfolded proteins and form a barrel shaped structure that protect, sequester nondisintegrated zone from the potential disoriented or tangled zone of proteins.

Cellular adaptive behavior with respect to stress may include retention of genetic stability or may relax the respective control pathways. These adaptive responses constitute intrinsic adaptive mechanism. During the initial stages the stressed cell tries to with stand the stressful conditions exhibiting short time adaptation. Functional aspects of a cell include cell adhesion, cellular spreading, cell division and inter and intra cellular communications. Further, in eukaryotes cellular arrangement, the tissues are designed in 3D geometries. Various functions of tissues and organs are facilitated due to homeotypic and heterotypic cellular interactions between parenchymal and non-parenchymal cells in tissues. During embryonic formative movements and development spatial and geometrical distribution of various cells, germinal layers, cell adhesion, cellular spreading follow the specific $3 \mathrm{D}$ design that is predetermined. The aggregate of one type of cells can be detached by fine agitation for short periods and improper washing while different types of cells can be set in to a specific 3D shape in a controlled manner. This aspect is very much employed during morphogenesis of embryos. The investigation on 3D spatial cellular organization is very helpful in understanding the dynamics of cancerous tissue, angiogenesis, tissue engineering or fabricating micro-tissues, regenerative medicine and drug designing [23].

\section{Significance}

Cells may be subjected to persistent stress because of the lifestyle, environmental conditions, use of therapeutic drugs, xenobiotics, age related problems and clinical applications like tissue transplantation, administration of biomedical devices etc. In the recent times most of these practices involve either directly or indirectly nanomaterials. During these practices quality and cellular well being are of paramount significance for the amicable successful treatment. Therefore it becomes obvious that understanding the mechanism involved during cellular stress and cellular response will enable to detect the derogative impacts and make an effort to reduce the intensity and frequency of developmental disorders due these conditions. It is essential to study the successful adaptation to stress to envisage the remedial aspects and development of relevant therapeutics, biomedical, biomolecular and clinical practices. This may further contribute to design the experimental model in fields like pharmacology, nanotheranostics and other related aspects to find the suitable and effective remedies. These may assist in fabrication and formulation of nanobased drug carriers, biomedical and nanobiomedical devices etc. Even environmental, exposure to consumer products resulting in risks or damages can be visualized and effective adaptive steps can be envisaged.

\section{Nanomaterials in the Service of Biological and Biomedical Fields}

Good number of nanomaterials like metallic, organic and inorganic nanoparticles, liposomes, polyplexes, quantum dots, carbon nanomaterials like carbon nanotubes, fullerene, graphene, etc, have been considered in the investigation related to biological, biomedical fields specifically also in the field of stem cell research [3,24-38] Synthetic materials like polylactides-co-glycolide (PGLA), polycaprolactone and naturally occurring materials like collagen, chitosan etc, have been involved in biological and biomedical research [23]. Some of the most common aspects that have been considered under stem cell research include non-evasive tracing of stem cells, transplanted progenitor cells, intracellular delivery of DNA, RNA interference molecules like proteins, peptides, genes, and small drugs either during stem cells differentiation or to investigate cellular biochemical processes [39]. These molecules are also utilized during the investigations related to the survival of the stem cells and biosensing of the physiological state of the stem cells [39]. When nanomaterials come in contact with a cell, cell organelles and biomolecules like proteins, nucleic acids or endoplasmic reticulum, mitochondria etc, a 'bionanointerface/nanobiointerface' is established at the site of contact between the two. The nature of bionanointerface depends on the colloidal forces, dynamic biophysicochemical forces and interactions involved. These forces result in various interactions that lead to the formation of protein corona, particle wrapping, inter and intracellular up-take, biocatalytic processes etc, depending and resulting in their biocompatibility, biodistribution and/or biodiversity. Further nanomaterials may undergo phase transformation, release of free energy, restructuring, dissolution etc at the interface [40]. The studies related to the interface formed provide useful information regarding the predictive designing of the structure and activity of nanomaterials [40]. Lancu, et al. are of the opinion that because of the unique features of the nanomaterials they behave like bioactive molecules. The potentials of nanomaterials and the totipotency/pluripotancy of stem cells in combination 


\section{Advances in Clinical Toxicology}

play a vital role in curing some of the incurable diseases like cancer, cirrhosis, injury to spinal cord, and neurodegenerative diseases etc [41].

Stem cells have a natural feature to get differentiated in to a special type of cells after genetic manipulation. Because of this feature the stem cells are considered as suitable tools for replacing cells in the field of regenerative medicine. The conventional modes to transfer gene to the progenitor cells are unsafe and exhibit lower degree of efficacy. Nanomaterials are a better option in this regard. Carbonate apatite with embedded fibronectin and E-cadherin chimera can conveniently acts as a carrier for functionalized DNA to the stem cells considered from the embryonic stage [42]. The transgenic delivery and expression are very distinct. Thus using functionalized nano-composites with DNA adhesive proteins can be a better option as a transfection system [42].

Nanomaterials such as peptides amphiphils, selfassembling peptides, nanotubes, layer-by-layer complexes, electron spun scaffolds, nanocomposites etc, have been very helpful and associated with the applied aspects of cell culture, encapsulation and as drug delivery agents. Nanomaterials are the preferred option because of their ability to get suitably modified or regulated even when within intracellular and extracellar environment. Further nanomaterials like DNA polyplexes and carbon nanomaterials etc act as good vehicles for cell delivery and also facilitate regenerative capacity. Such nanomaterials can be conveniently characterized as per the clinical safety and therapeutic applications [43]. Chemically fabricated gold coated collagen nanofibers are biocompatible and have the ability to elevate the degree of cellular differentiation of mesenchymal stem cells in to myocardial and neuronal cells. This process of differentiation is associated with the expression of markers related to atrium, natriuratic peptide, actin-F, actin monomer, glial acidic protein and neurofilaments [44].

In recent times, different nanomaterials have been experimented on in the fields of biological, biomedical and pharmacological/pharmaceutical area etc. A condition in which $\alpha$-galactosidase activity is absent results in the accumulation of glycosphingolipids in vasculature [45]. This condition in turn can cause multiple organ pathology. Such pathological conditions may be rectified by the enzyme replacement therapy. This therapy has many deficiencies and a lower degree of efficacy. Such conditions are corrected and correlated by intracellular penetration of human recombinant $\alpha$-galactosidase- $A$ enzyme loaded within nanoliposomes that are functionalized with arginine-glycine-aspartate peptide. Such protein-nanoliposomes conjugates act as bioactive nanomaterial and have therapeutic benefits [45].

Fabricated graphene quantum dots are $\mathrm{pH}$ sensitive carriers for chemotherapeutic drugs. The drug is released as a result of acidification of vesicle in cells. Cancer cells uptake these carriers conveniently when functionalized with RGD (arginine-glycine-aspartate peptide). The graphene quantum dots are better options as they reach cancer cells readily [46].

Single walled carbon nanotubes (SWCNT) covalently bonded with radio metal ion chelates (DOTA) and desferrioxamine (DFO) were used in the study with reference to the activity and clearance from blood [47]. It was seen that pharmacokinetics related to these fabricated SWCNT could influence the blood clearance and facilitated distinct imaging. The tomography of the tumor was imaged using 'Near infrared' 3D fluorescence technique. During this study it was seen that SWCNT construct was safe and murine cells model could tolerate it comfortably. This indicates that the SWCNT based nanomaterials are a favorable option to be implicated in tumor vascular tissues studies [47]. Multiwalled carbon nanotubes (300 and $700 \mathrm{~nm}$ in length) were functionalized with Poly (4-polyvinvylpyridine). Their uptake and translocation was observed in case of pulmonary respiratory epithelial barrier. The monoculture of immortalized human alveolar epithelial type cell and primary human alveolar epithelial type cell were used as test models. The rate of uptake and translocation of these functionalized multiwalled carbon nanotubes was different in these test cells and was dependent on the physicochemical properties of nanoparticles and the nature of cell. The degree of multiwalled carbon nanotubes uptake with smaller length (300 nm) was higher than that of multiwalled carbon nanotubes with larger length $(700 \mathrm{~nm})$. Even the translocation rates were different in the cell-type studied [48].

Fullerene $\mathrm{C}_{60}$ along with its water soluble forms have the potential to protect against damage either biochemical or specifically oxidative stress [33]. Graphene sheets are modified in to nana-porous silica coated material and specific target peptides can be conjugated. The resultant product is suitable for carrying cancer treating drugs and its regulated release. The release of loaded chemicals is based on the factors like heat and $\mathrm{pH}$ [2]. DNA nanomaterial has become very handy because DNA molecule has the precise structure, very high degree 
of biocompatibility, varied functionality and ability to undergo self assembly. These features of DNA make it one of the most suitable tools as a carrier and applications in biological, biomedical and therapeutic applications [49]. DNA as nanomaterials exhibits very high degree of biocompatibility and cellular up take. It can be modified in to DNA nanotubes, DNA tetrahedral and origami nanorobots; these forms are very useful in the field of drugs, therapeutics and siRNA based gene silencing techniques [50]. Nanoparticles such as silica, gold, titanium dioxide, silver and iron oxide also play significant roles in modulating stem cell differentiation [51].

\section{Nanobio-Interface and its Role in Cellular Behavior}

Nanomaterials are considered to be similar to endogenous biomolecules because of their ability to move across most of the biological barriers, and to react with bio-molecules [2]. As mentioned above 'nanobiointerface' is established between nanomaterials and biomolecules within bioenvironment. This interface represents how the biomolecules react or 'see' the nanomaterial and vice versa. The result of this interaction reflects on the attachment/binding of biomolecule on the surface of nanomaterial as if the biomolecules are attached to the scaffolds made of nanomaterials. If this nanobiointerface is suitably and intelligently designed it can help to understand the functionality of the nanoscaled system in biological environment. Further, the mechanism involved at nanobiointerface formed between nanomaterials like nucleic acids, polymers, peptides, proteins, antibodies etc, can be elaborated. Therefore the application of nanobiointerface formation may be utilized in probing varied biological processes [52]. Cellular adhesion is another aspect of cellular behavior. This aspect is dependent on the features of the substrate like chemistry, topography, hydrophobicity and surface energy etc, exhibited by the interacting extracellular matrix or cells. To ensure a specific set target the substrate can be designed with reference to the regenerative medicine and tissue engineering applications [53]. Nanobiointerface exhibits some of the controllable topographic features like nanoparticle interface, nano- pattern, combined nano and microtopography, nano structures as natural and polymeric complex mixed composites etc [54].

Topographic aspect of nanomaterials and the interacting cells is a very important aspect in the fields of applied nanotechnology and biomedical investigations. The topographical characters have substantial impacts on the cellular behavior with respect to the interface and surface interactions resulting in some of the independent biochemical indications [54]. Cellular functions and behavior like cellular adhesion, inter and intracellular communication, cellular differentiation etc, are dependent on the cellular and extracellular matrix and their topographic nature. Nanoscaled engineered materials can be fabricated in to an artificial extracellular matrix that provides a combined impact of physical, chemical, mechanical and biological features. Over all resultant impact of these nanoscaled materials facilitates the cellular functionality specifically for inter and intra cellular communication [54]. There exist nanoscaled spaces between extracellular matrix and cell ligands, integrin distribution, and signal transduction components of the cell. When nanomaterials having specific geometrically defined and biofunctionalized surface are brought in contact with the stem cells these get influenced. Thus this improvised extracellular matrix is able to induce stem cells to carry out their natural behavior [55]. Stem cells exhibit multipotency when present in their respective natural 'biological niche' but lose this behavioral ability when the stem cells are subjected to culture medium. With this their lost behavioral ability, their clinical and biomedical applications also become debatable. If engineered artificial analog to their respective biological natural niche' are employed in cell cultural experiments then the biofunctionality can be restored. A macroporous hydrogel scaffold fabricated resembling sponge architectural trabacular bone can effectively retain the respective biofunctionality of hematopoietic stem cells. Even mesenchymal and non-mesenchymal cells from umbilical cord, bone marrow and osteoblasts have shown biofunctional behavior when co-cultured in pores of hydrogel scaffold. Thus this nanofabrication can be a good biomimatic medium to investigate the new clinical and biomedical applications of the nanomaterials [56].

The properties of surface of nanoscale materials affect varied molecular and biological processes at the site of bionanointerface harnessing recent modes of designing materials to be used in the field of biomedical devises. Nanotechnology, materials engineering, nanoengineering are being used as tools in predicting, diagnosing means in biomedical field [57]. Cell adhesion with the extracellular matrix is influenced by the specific class of integrin transmembrane receptors. A peptide sequence that is made of three amino acids namely arginine, glycine and aspartic acid plays a significant role in identifying the related integrin to ensure cell adhesion. Even the density and lateral distribution of ligand associated with cell adhesion is one of the parameters that affect this process. 
Conditions like reduced number of adhering cells, declined or restricted area of cells to spread (upper limit of space is below $58 \mathrm{~nm}$ ) and restricted forces for adhesion are responsible for the need of cluster of $\alpha_{v} \beta_{3}$ integrin to obtain appropriate formation of the contact. Nanopatterned interface can be utilized to investigate and mimicking the cell to cell contact [58].

The study related to cell adhesion, differentiation and proliferation gets influenced by the interactions between fabricated materials/and nanomaterials and cells/tissues [58]. The parameters involved in these interactions may be used as a guide to design suitable options depending on the physicochemical features of the material involved. Some the parameters include hydrophobicity and the charge on the materials. If the material used is moderately hydrophilic and positive charged the cells exhibit moderate adhesion [59]. The cell adherent molecules such as vitronectin and fibronectin get adsorbed on such substrate. This cell adhesion takes place in a specific geometric formation [60]. This geometrical formation is dependent on the adhering associated molecules as these can be available to cell adhesion receptors i.e. integrins. The degree of hydrophilicity is an important parameter because higher degree of hydrophilicity restricts adhesion of these proteins because possibly these are bound with weak bonds. Surface with higher degree of hydrophobicity is also not favorable for cell adhesion. This process is hampered if the proteins are adsorbed rigidly and/or are in denatured form. Other parameters like wettability, physical treatment by irradiation with ions, plasma or UV radiation influence cell adhesion specifically if synthetic polymers are used as substrate. The synthetic polymerized substrate when activated by irradiation and functionalized using varied biomolecules specific nanomaterials can enhance the cell functional/behavioral efficacy. Roughness and surface topography also affect the cell adhesion. Nanostructures having irregularities (dimensions $100 \mathrm{~nm}$ ) as substrate are most suitable for cell adhesion and growth but similar material with micro sized irregularities are not suitable for induction of cell adhesion. These structures with micro sized irregularities hamper cell spreading, cell proliferation and cell differentiation. Cells induce traction forces due to their adhesion process. Because of these forces cell do not attach, spread, and survive on the highly soft and deformable substrate. Surface of substrate having specific nanopattern promotes the zone selective cell adhesion and growth. These features can be favorable options in the field of tissue engineering [61].

Gold nanoparticles have been utilized in biological and biomedical fields as labeling, delivering, sensing and heating agents $[28,29,62]$. Tissue engineering and regenerative medicine are focused to develop a suitable substitute that ensures restoration, maintenance, and improves the damaged tissues structurally and functionally. Nanotechnology is playing its significant role in many fields and tissue engineering and regenerative medicine are also being supported by its products. Nanomaterials offer broad scope for the suitable materials to be used as substitute. This is because of their unique physicochemical features and the ease with which these can be modified. Most of the nanomaterials are biocompatible and cytocompatible in comparison to the microsized materials. Tissues like bone, cartilage, vascular, neural and urinary bladder tissues have shown good results with respect to their maintenance, growth and functionality [60]. Gold nanoparticle (60nm) enhanced cell proliferation in case of human periodontal ligament cell in vitro. These nanoparticles also enhanced cell differentiation in case of osteoblast cells but did not influence the cell differentiation in case of adipogenic cells. The cell differentiation process for osteoblasts and their functions are regulated by transcription factors. The related factors are collagen type-1 (Col-1), Raunt related transcription factor-2 (Runx-2) bone sialoprotein (BSP) and osteocalcin (OCN). The expression of these transcription factors are also influenced by gold nanoparticles [63].

Role of protein during cellular adhesion is well evident and so is the adherence of cell to protein resistant surface. Modified gold nanoparticles as 3D topographical gold nanoparticle layer by chemical plating were fabricated. Other set of gold nanoparticles were considered as smooth nano form. Both forms were subjected to study cell adhesion comparatively. More cells adhered to topographically fabricated gold nanoparticles in comparison to the non-topographically modified structures. The degree of cell adherence was firm and quantitatively more on topographically modified gold nanoparticles. The cells that adhered to these nanoparticles developed lamlipodia and filopodia [59].

The fate of cell under stress depends on the type, duration, exogenous parameters and cellular ability to survive. Stressed Cells exhibit some form of interplay after which the decision is made either for survival or death. These cells may undergo death involving any of the processes like apoptosis, necrosis, autophagic cellular death or pyroptosis and autophagy. The implication of medical aspects of cellular stress may induce health issues, pathogenesis, like diabetes, neurodegenerative diseases like Parkinson's disease, myocardial infarction, cancer, and untimely senescence $[64,65]$. 
Nanomaterials are among the most suitable options as diagnostic tools because these can be conveniently formulated to be used appropriately as theranostics for the treatment of ailments. Specifically designed nanoparticles have the ability to identify, reach and interact with the target cells or tissues causing minimum derogative impacts. These specifically fabricated nanomaterials reach the site of injury and provide desired profile of drug release. Nanomaterials have features like surface plasmon resonance, specific high surface reactive area, magnetism, the ability to get structurally modified and ability to move across most of the biological barriers [2]. These little wonders become better alternatives in comparison to the traditional diagnostic and therapeutic techniques [66].

Nanoparticles show physicochemical features and ability to get modified as per the requirements for specific applications. These nanomaterials may be carbon based, organic, and inorganic in nature. These are applied in medical, biomedical, biotechnological, biomolecular, materials science, tissue engineering, DNA technological, enzyme immobilization and pharmacological fields. Liposomes and polymer are organic nanomaterials used as drug and biomolecule carriers. Metallic nanoparticles are potential options for imaging techniques as contrast agents and formulations for conjugate ligand. Metal oxide nanoparticles are preferably used during antioxidative and catalytic activities, drug delivery and as biosensors. Ceramics and quantum dots are used as drug vehicles, bioimaging and biosensors. Fullerene and carbon nanotubes are suitable as imaging agents [67].

Liposomes are effective agents for drug delivery specifically during the treatment of cancer. These are used to carry bioactive substances like peptides, enzymes, therapeutic drugs and food ingredients. Such nanomaterials are biocompatible and biodegradable [68,69]. Polymeric nanomaterials are biodegradable and biocompatible hence are very useful agents for drug delivery. Natural polymers like chitosan, synthetic polymers such as polylactides, polymethylacrylate, and polyethylene glycol etc are used in biomedical and pharmaceutical fields. Their surfaces can be readily modified suitably and sized so that their solubility, dispersibility can be regulated [70].

Metal oxide nanoparticles like titanium di oxide $\left(\mathrm{TiO}_{2}\right)$, iron oxide $\left(\mathrm{Fe}_{3} \mathrm{O}_{4}\right)$, zirconium $\left(\mathrm{ZrO}_{2}\right)$, Cerium oxide (Ceria) $\left(\mathrm{CeO}_{2}\right)$ are being used as in biomedical and pharmaceutical fields because these metal oxides are efficient catalytic agents and possess good antioxidant capacity. Doped zinc oxide nanoparticles have potential to reduce inflammatory oxidation and also act as effective agent for bio-imaging [71]. Cerium oxide $\left(\mathrm{CeO}_{2}\right)$ is good autocatalyst and has effective antioxidant properties. It also acts as a useful cell labeling agent for MRI contrast imaging. Such nanomaterials are good options as remedy to treat spinal injury, stroke and degenerative retinal conditions [72]. Magnetic iron oxide nanoparticles, supraparamagnetic iron oxide, small supraparamagnetic iron oxide are being used because of their 'longer blood shelf-life'. These are also preferred as contrast agent for MIR techniques to investigate atherosclerotic plaque. Other nanoparticles used for MRI are lipid-encapsulated perfluorocarbon emulsion' with godalium and nanoparticles having high density lipoprotein.

Ceramic nanoparticles are inorganic porous materials. Some of them are most suitable as carriers for drugs, proteins, peptides and enzyme immobilization. This is because their porosity is not compromised due to either swelling or impact of temperature or $\mathrm{pH}$ of their ambient environment. $\mathrm{CeO}_{2}$ is one of the common ceramic nanomaterials that is used as capped mesoporous silica nanomaterial as drug carrier for treating lung cancer. Other ceramic nanomaterials are clay minerals, cement, glass, bio-ceramics etc. These are used in dental, orthopedics, material science, tissue engineering and other biomedical applications. Calcium phosphate, calcium sulfate and carbonate, tricalcium phosphate, hydroxyapatite, tricalcium phosphate hydroxyapatite, bioreactive glass, bioreactive glass ceramic, titania based ceramic, alumina ceramics, zirconia and ceramic polymers composite are the examples of ceramic nanomaterials being used in nanotechnology. These ceramic nanomaterials are extensively used in orthopedics, regeneration of bone, tissue development, tissue engineering, nanomedicine and other biomedical applications.

Quantum dots are made of semiconductor matter with fluorescent features and or photo-luminescent properties. Their core is made of semiconductor matter. Some of the common quantum dots are cadmium/selenium $(\mathrm{Cd} / \mathrm{Se})$, cadmium/tellurium ( $\mathrm{Cd} / \mathrm{Te}$ ), indium phosphate (In/P), indium arsenate (In/As) etc. Some of the quantum dots have outer coat (shell) of zinc sulfide that acts as a protective measure against leakage of toxic heavy metal. This also elevates their optical and physical features so that these are suitably used in bioimaging and bioassay techniques. These nanomaterials exhibit better functionality in comparison to the organic fluorescent dyes. The quantum dots interact with biomolecules conjugating with them specifically when active groups like carboxyl, thiol, epoxy, hydroxyl and aldehydes are 
bound on to their surface. Conjugation between quantum dots and biomolecules is dependent on factors like size, surface properties of quantum dots. The conjugation process is selectively specific with respect to varied applications [73]. Quantum dots also get conjugated with biomolecules like proteins, peptides, oligonucleotides. These nanomaterials in conjugated form enhance their chances of binding with desired or designated sites. Gold quantum dots possess colorimetric properties but not fluorescent properties. These quantum dots exhibit enhanced surface plasma resonance related to shape, size, solvency (dissolvability), ligand and functionalization of their surface. These nanomaterials also have dielectric properties and tendency of agglomeration. Because of these unique features quantum dots are among preferred options for detection of DNA sequencing, hybridization assay, genetic disorders, immunoblot assay etc. Fullerene and carbon nanotubes are carbon based nanomaterials that are commonly preferred options for biomedical applications. Generally pristine carbon nanotubes are likely to be toxic functionally but if their surface chemistry is functionalized or modified these nanoparticles become suitable to be used in pharmacological applications. When functional groups like $-\mathrm{COOH},-\mathrm{OH}$ and $-\mathrm{CO}$ are added on to the surface of the formulated carbon nanotubes these are either less toxic or behave in non-toxic manner. The ultra short single walled nanotubes (SWCNT) are used as non-viral vectors. These are effective carriers for oligonucleotides molecules for their applications in the fields of biomolecular and biomedical sciences. Non-covalent interactions of carbon nanotubes are biocompatible hence these are used in biomolecular, biomedical and biotechnological fields (Figure 1).

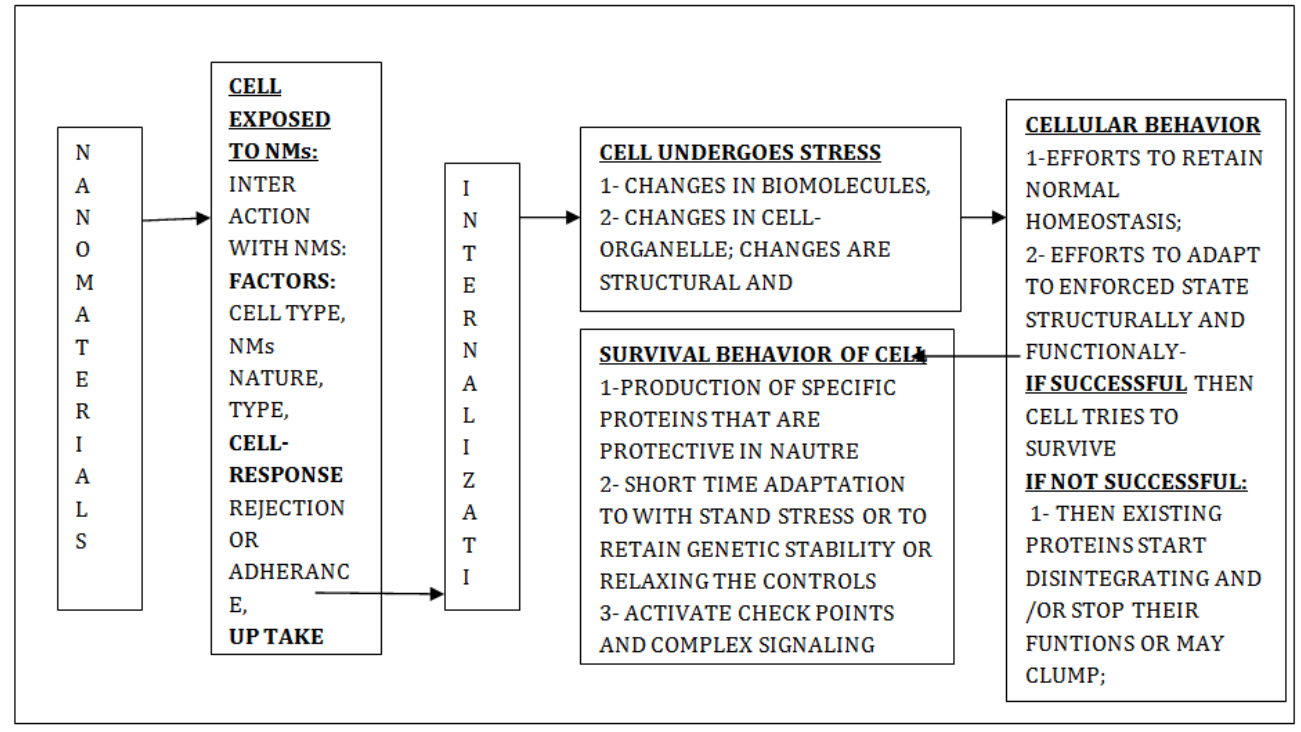

Figure1: Graphic Representaion of Influence of Nanomaterials (Nms) On Cell.

The investigations on human genome sequencing indicate that around $1.5 \%$ of DNA sequence codes for proteins and major portion (98.5\%) is related to code for non-coding RNAs. This observation suggests that there are options to formulate theranostics that involve RNA targeting drugs and RNA drugs. This option is in addition to chemical and protein drugs. Currently an effort is being made to use stable RNA as a potential option to formulate drug for the treatment of cancer. Theranostics related to RNA are very special because the scaffold, ligands, and the components of theranostics primarily involve RNA. Their physicochemical features facilitate appropriate delivery of
siRNA, miRNA, ribozyme or ribowitches. Some of the imaging techniques involve fluorogenic RNA and RNA aptamer. These help the theranostics to reach the specific target [75-77]. Thus, nanotechnology is an open field to investigate and study the mechanism of cell stress caused due to nanomaterials with reference to the medical implications.

\section{Conclusion}

The stress in bio-system is due to physical forces, deficient resources, infection, toxins, change in cellular 
ambient environment and some undefined stressors. During cellular stress there is increased degree of fluctuations in the metabolic aspects of DNA, RNA, protein signaling pathways etc. Cells under stress exhibit adaptive behavior or sustenance behavior. If a cell fails then it may undergo derogative changes related to proteins, senescence, (permanent rest), and/or trigger cell death. Generally stress is the impact of some of the factors like molecular flux, flow and change in body fluid, intrinsic and extrinsic cellular movements, change in temperature etc. This aspect is of significance in biological, biomedical, tissue engineering and tissue replacement therapy. Nanotechnology is playing a significant role in many fields. Tissue engineering and regenerative medicine are no exception to this technology. Nanomaterials offer broad scope for the suitable materials to be used as substitutes. Cell culture plays very significant role for applications in tissue engineering. Nanotechnology and nanomaterials have enormous potential to either correct or improve the efficacy of the existing tissue technology and tissue engineering. Cell culture and stem cells technologies have natural features that facilitate transformation of special type of cells and other aspect like genetic manipulation, and other interactions in relation to nanomaterials. Stem cells are considered as a suitable tool for replacing cells technique in the field of regenerative medicine. The conventional modes to transfer gene to the progenitor cells are unsafe and exhibit lower degree of efficacy. Nanomaterials are a better option in these technologies with intentions of more experimentation to fill the existing lacunae.

Declaration: Authors declare that there is no conflict of interest.

Contribution: All authors contributed equally. Authors have revised the manuscript. Authors have agreed with the concept presented and give consent for its publication.

Acknowledgement: There is no acknowledgement.

Funding: No funding was involved during this presentation.

\section{References}

1. Lahir YK (2016) Application of time and scale concept in biological systems. Biochemical and Cellular Archives 16(2): 231-237.
2. Lahir YK (2014) Morphological aspects of intercellular communication. Bionano Frontier 7(1): 3-11.

3. Lahir YK (2018) Impacts of metal and metal oxide nanoparticles on reproductive tissues and Spermatogenesis. J Experimental Zoology 18(2).

4. Mc Graw (2003) Hill directory of scientific and technical terms, 6E. The McGraw Hill C Inc.

5. Kultz D (2003) Evolution of cellular stress proteasome: from monophyletic origin to ubiquitous function. The Journal of Experimental Biology 206(Pt 18): 3119-3124.

6. Bulaven DV, Amundson SA, Fornace AJ (2002) p38 and Chk-1 kinases: different conductors for G2/M check point symphony. Curr Opin Genet Dev 12(1): 92-97.

7. Kato I, Ito H, Iwamato I, Lida K, Inaguma Y (2001) Protein kinase inhibition can suppress stress induced dissociation of Hsp 27. Cell Stress Chaperones 6(1): 16-20.

8. Vermeulen L, De Wilde G, Notebaert S, Berghe WV, Haegeman G (2002) Regulation of transcriptional activity of the nuclear factor kappa $\beta$ subunit. Biochem Pharmacol 64: 963-970.

9. Jung T, Catalogol B, Grune $T$ (2009) The proteasomal system. Mol Aspects Med 30(40): 191-296.

10. Kourtis N, Tavernarakis N (2011) Cellular stress response path ways and aging: intricate molecular Relationship. EMBO J 30(13): 2520-2531.

11. Leestemaker Y, Ovaa H (2017) Tool to investigate the ubiquitin proteasome system. Drug Discov Today Technol 26: 25-31.

12. Milisav I (2011) Cellular Stress Responses, Advances in Regenerative Medicine, In; Sabine WisletGendebien (Eds.), In Tech, Rijek, Croatia Shanghai, China, pp: 20.

13. Moor MA (2001) The role of chemo-attraction in cancer metastasis. Bioassay 23(8): 674-676.

14. Lo CM, Wang HB, Dembo M, Wang YL (2000) Cell movement is guided by the rigidity of Substrate. The Biophys J 79(1): 144-152. 


\section{Advances in Clinical Toxicology}

15. Balkwill F (2004) Cancer and chemokine net work. Nat Rev Cancer 4(7): 540-550.

16. Wang JC, Li X, Lin B, Shim S, Ming G, et al. (2008) A micro fluidics based turning assay, Reveals complex growth cone response to integrated gradients of substrate-bound extracellular matrix molecules and diffusible guidance cues. Lab Chip 8(2): 227-237.

17. Cheong R, Paliwal S, Levchanko A (2010) Models at single cell level, Wiley Interdisciplinary Reviews: Systems Biology and Medicine 2(1): 34-48.

18. Online Mendelian Inheritance in Man (OMIM) (2011) Johns Hopkins University, Baltimore.

19. Speidel D (2010) Transcription-independent p53 apoptosis: an alternative route to death. Trends Cell Biol 20(1): 14-24.

20. Ak P, Levine AJ (2010) p53 and NF-kB: different strategies for responding to stress lead to a functional antagonism. FASEB J 24(10): 3643-3652.

21. Nipic D, Pirc A, Banic B, Suput D, Milisav I (2010) Preapoptotic cell stress response of primary Hepatocytes. Hepatology 51(6): 2140-2151.

22. Banič, B, Nipič D, Suput D, Milisav I (2011) DMSO modulates the pathway of apoptosis triggering, Cell Mol Biol Lett 16(2): 328-341.

23. Tekin H, Shanchez JG, Landeros C, Dubbin K, Langer R, et al. (2012) Controlling spatial organization of multiple cell types in defined geometries. Adv Mater 24(41): 5543-5555.

24. Lam T, Avit PK, Pouliot P, Tardif JC, Rheaume E, et al. (2016a) Magnetic resonance imaging/inflorescence dual modality protocol using designed phosphate ligand coupled to super paramagnetic iron oxide nanoparticles. The Journal of Materials Chemistry B 4(22): 3969-3981.

25. Lam T, Avit PK, Pouliot P (2016b) Surface engineering of SPIONs role of phosphate ligand multi valency in tailoring their efficiency. Nanotechnology 27(41): 415-602.

26. Lam T, Pouliot P, Tardif JC, Avti PK, Maafi F, et al. (2016c) Fabricating water dispersible super paramagnetic iron oxide nanoparticles for biomedical applications through ligand exchange and direct conjugation. Nanomaterials 6(6): 100.
27. Lam T, Pouliot P, Avit PK, Lesage F, Kakkar AK (2013) Supraparamagnetic iron oxide nanoprobes for imaging and theranostics. Advances in Colloid and Interface Science 199-200: 95-113.

28. Bedard M, Avit PK, Lam T, Rouleau L, Tardif JC (2015) Conjugation of multivalent ligand to gold nanoshells and designing a dual modality imaging Probe. Journal of Materials Chemistry 3(9): 1788-1800.

29. Ng VWK, Avit PK, Bedard M (2014) Mictoarm conjugated multifunctional gold nanoshells: synthesis and evaluation of biocompatibility and cellular uptake. Journal of Material Chemistry 2: 634-6344.

30. Avit PK, Maysinger D, Kakkar A (2013a) Alkyne-azide 'click' chemistry in designing nanocarriers for applications in biology. Molecules 18(8): 9531-9549.

31. Sitharaman B, Avit PK, Schaefer K, Talukdar Y, Longtin JP (2011) A novel nanoparticle-enhanced photoacoustic stimulus for bone tissue engineering. Tissue Eng Part A 17(13-14): 1851-1858.

32. Sitharaman B, Rajamani S, Avit PK (2011) Time resolved red luminescence from europium catalyzed single walled carbon nanotubes. Chem Commun (Camb) 47(5): 1607-1609.

33. Lahir YK (2017) Impacts of fullerene on biological system. Clinical Immunology Endocrinology and metabolic Drugs 4(1): 48-57.

34. Takuldar Y, Avit PK, Sun J, Sitharaman B (2014) Multimodal ultra sound photo acoustic imaging of Tissue engineering scaffolds and blood oxygen saturation in and around the scaffolds. Tissue Eng Part C Methods 20(5): 440-449.

35. Avit PK, Caparelli ED, Sitharaman B (2013b) Cytotoxicity, cytocompatibility, cell labeling efficiency and in vitro cellular magnetic resonance imaging of gadolinium-catalyzed single walled carbon nanotubes. J Biomed Mater Res A 101(12): 35803591.

36. Avit PK, Talukdar Y, Sirotkin MV, Shroyer KR, Sitharaman B (2013c) Toward single walled carbon nanotube gadolinium complex as advanced MIR contrast agents: pharmcodynamics and global genomic response in small animals. J Biomed Mater Res B Appl Biomater 101(6): 1039-1049. 
37. Avit PK, Sitharaman B (2012a) Luminescent single walled carbon nanotube sensitized europium Nanoprobes for cellular imaging. Int J Nanomedicine 7: 1953-1964.

38. Avit PK, Hu S, Favazza C, Mikos AG, Jansen JA, et al. (2012b) Detection and mapping and quantification of single walled carbon nanotubes in histological Specimens with photo-acoustic microscopy. PLOS One 7(4): e35064.

39. Ilie I, Ilie R, Mocan T, Bartos D, Mocan L (2012) Influence of nanomaterials on stem cells differentiation: Designing an appropriate nanobiointerface. Int J Nanomedicine (7): 2211-2225.

40. Nel AE, Madler L, Velegol D, Xia T, Hoek EMV, et al. (2009) Understanding biophysicochemical interactions at the nanobiointerface. Nature Materials 8(7): 543-557.

41. Lancu C, Ilie R, Georgescu CE, Ilie R, Biris AR, et al, (2009) Applications of nanomaterials in cell stem therapies And onset of nano medicine. Particulate Science and Technology 27(6): 562-574.

42. Kutsuzawa K, Akaike T, Chowdhury EH (2008) Influence of the cell adhesive protein E-cadherin and fibronectin embedded in carbonate-apatite DNA carrier on transgene delivery and expression in a mouse embryonic stem cell line. Biomaterials 29(3): 370-376.

43. Wan AC, Ying JY (2010) Nanomaterials for in situ cell delivery and tissue regeneration. Advanced Drug Delivery Reviews 62(7-8): 731-740.

44. Orza A, Soritau O, Olenic L, Diudea M, Florea A, et al. (2011) Electrically conductive gold coated collagen nanofibers for placenta derived mesenchymal stem cells enhanced differentiation and proliferation. ACS Nano 5(6): 4490-4503.

45. Cabrera I, Abasolo I, Corchero JL, Elizondo E, Gil PR, et al. (2016) $\alpha$-galactosidase-A loaded nanoliposomes with enhanced, enzymatic activity and intracellular penetration. Advance Health Mater 5(7): 829-840.

46. Qiu J, Zhang R, Li J, Sang Y, Tang W, et al. (2015) Fluorescent graphene quantum dots as taceable, $\mathrm{pH}$ sensitivedrug delivery system. Int J Nanomedicine 28(10): 6709-6724.
47. Ruggiero A, Villa CH, Holland JP, Sprinkle SR, May C, et al. (2010) Imaging and treating tumor vasculature with targeted radio-labeled carbon nanotubes. Int J Nanomedicine 5(5): 783-802.

48. Ruenraroengsak $\mathrm{P}$, Chen $\mathrm{S}, \mathrm{Hu} \mathrm{S}$, Melbourne J, Sweeney S, et al. (2016) Translocation of functionalized multi-walled carbon Nanotubes across human pulmonary alveolar epithelium: dominant role of epithelial type 1 cells. American Chemical Society Nano 10: 5070-5085.

49. Ouang X, Chao J, Su S (2017) DNA nanostructure as smart carrier or drug delivery, In Ke Y (Eds.), 3D DNA nanostructure, Methods in Molecular Biology, Humana Press, New York, USA.

50. Li j, Fan C, Pei H, Shi J, Huang Q (2013) Smart drug delivery nanocarriers with self assembled DNA nano structure. Advanced Materials 25(32): 4386-4396.

51. Wei M, Li S, Le W (2017) Nanomaterials modulate stem cell differentiation: biological interaction and underlying mechanisms. J of Nanobiotechnology 15: 75.

52. Kah JC, Yeo EL, Koh WL, Poinard BE, Neo DJ (2013) Nanoparticles interface to biology: Applications in probing and modulating biological processes. Crit Rev Biomed Eng 41(4-5): 323-341.

53. La-Pointe VLS, Fernandes AT, Bell NC, Stellacci F, Stevens MM (2013) Nanoscale topography and chemistry effect embryonic stem cell self-renewal and early differentiation Advanced Healthcare Materials 2(12): 1644-1650.

54. Liu XQ, Tang RZ (2017) Biological responses to nanomaterials understanding nanobio effects oncell behavior. Drug Deliv 24(2): 1-15.

55. Altrock E, Muth CA, Klein G, Spatzab JP, Thediecka CL (2012) The significance of integrin/ ligand nanopatterning on lipid raft clustering in hematopoietic stem cells. Biomaterials 33(11): 31073118.

56. Raic A, Rodling L, Kalbacher H (2014) Biomimetic macroporous PEG hydrogel as 3D scaffolds for multiplication of human hematopoietic stem and progenitor cells. Biomaterials 35(3): 929-940.

57. Variola F, Bruski JB, Orsini G, Tambasco de Oliveira P, Wazen R, et al. (2011) Nanoscale surface 
modifications of medically relevant metals: state of the art and perspectives. Nanoscale 3(2): 335-353.

58. Lohmuller T, Aydin D, Schwieder M, Morhard C, Louban I, et al. (2011) Nanopatterning by block copolymer micelle nanolithography and bioinspired applications. Biointerfaces 6(1): MR1-MR12.

59. Shi X, Wang Y, Li D, Yuan L, Zhou F, et al. (2012) Cell adhesion on POEG MA-modified topographical surface. Langmuir 28(49): 17011-17018.

60. Zhang L, Webster TJ (2009) Nanotechnology and nanomaterials. Promises for improved tissue regeneration. Nano Today 4(1): 66-80.

61. Bacakova L, Filova E, Parizek M, Ruml T, Svorcik V (2011) Modulation of cell adhesion, Proliferation and differentiation on materials designed for body implants. Biotechnolo Adv 29(6): 739-767.

62. Sperling RA, Gil PR, Zhang F (2008) Biological applications of gold nanoparticles. Chem Soc Review 37(9): 1896-1908.

63. Li C, Li Z, Wang Y, Liu H (2016) Gold nanoparticles promote proliferation of human periodontal ligament stem cells and have limited effects on cell differentiation. Journal of Nanomaterials 2016: 10.

64. Poljsak B, Milisav I (2011) Clinical implications of cellular stress response. Bosn J Basic Med Sci 12(2): 122-126.

65. Godey LD, Rossgnoli MI, Poliana DP, Norbetro GC, Eduardo H de LU (2018) A comprehensive Overview on stress neurobiology: basic concept and clinical implications. Front Behav Neurosci 3(12): 127.

66. Wang YJ, Larsson M, Huang WT, Chiou SH, Nicholls SJ, et al. (2016) The use of polymer based nanoparticles and nanostructured materials in treatment and diagnosis of cardiovascular diseases: Recent advances and emerging design. Progress in Polymer Science 57: 153-178.

67. Mauricio MD, Guerra-Ojeda S, Marchio P, Valles SL, Aldasoro M, et al. (2018) Nanoparticles in medicine: A focus on vascular oxidative stress. Oxidative medicine and Cellular Longevity 2018: 20.

68. Malam Y, Loizidou M, Seifalian AM (2009) Liposomes and nanoparticle: nanosized vehicles for drug delivery in cancer. Trends in Pharmaceutical Science 30(11): 592-599.

69. Panachi Y, Masound F, Majid M, Mirahadi M, Khalilov $\mathrm{R}$, et al. (2017) Recent Advances on liposomal nanoparticles. Artificial cells Nanomedicine and Biotechnology 45(4): 788-799.

70. Khalid M, El-Sawy HS (2017) Polymeric nanoparticles: promising platform for drug delivery. Int J Pharm 528(1-2): 675-691.

71. Anrecscu S, Ornatska M, Erlichman JS, Estevez A, Leitter JC (2012) Biomedical applications of metal oxide nanoparticles. Fine particles in Medicines and Pharmacy, pp: 57-100.

72. Celardo T, Pedrerson JS, Traversa E, Ghibelli L (2011) Pharmacological products of cerium oxide and nanoparticles, Nanoscale 3(4): 1411-1420.

73. Bartek J, Inkas J (2001) Pathways governing G1/S transition and their response to DNA damage. FEBS Lett 490(3): 117-122.

74. Foubert A, Belogazova N V,Rajkovic A, Sas B, Madder A, et al. (2016) Bioconjugation of quantum dots: review and impact on future applications. TrAc Trends in Analytical Chemistry 83: 31-48.

75. Shu Y, Fengmei P, Sharma A, Rajabi M, Haque F, et al. (2014) Stable RNA nanoparticles as potential new generation drug for cancer therapy. Adv Drug Deliv Rev 66: 74-89.

76. Harkin DP, Hall PA (2000) Measuring a cell's response to stress: the p53 pathway. Genome Biology 1(1): R105.

77. Kristie S, Emily V (2012) Cell stress, National Institute of Health.

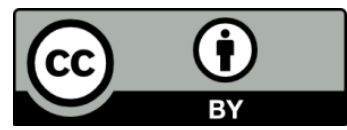

